\title{
المهارات الأدائية في مقرر التصميم الجر افيكي للوسـائط المرئية في تخصص تكنولوجيا التعليم لمرحلة البكالوريوس في جامعة الشرق الأوسط ومدى تطبيق الطلاب لها من وجهاة نظرهم
}

\author{
لينا راسم عرفة \\ معلمة حاسوب- لواء وادي السير - التعليم الخاص- المملكة الأردنية الهاشمية \\ lina3arafah84@gmail.com
}

DOI: https://doi.org/DOI:10.31559/EPS2020.7.3.6

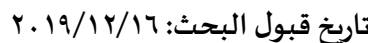

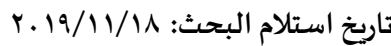

هدفت هذه الدراسـة إلى تحديد المهارات الأدائية (العملية) في مقرر التصيميم الجرافيكي للوسائط المرئية في تخصص تكنولوجيا التعليم لدرجة البكالوريوس في جامعة الشرق الأوسط باستخدام أداة تحليل المحتوى، وتحديد مدى تطبيق الطلبة للمهارات الأدائية (العملية) التي يتضمنها المقرر و

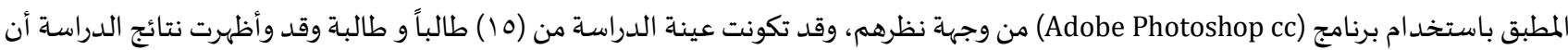

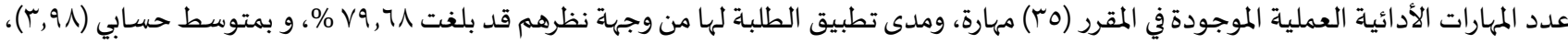
وتصنيف تطبيق بدرجة عالية، وتوصي الباحثة بالتركيز أكثر على تفعيل وربط البرمجيات الحاسوبية بتصميم وإنتاج المحتوى التعليمي الإلكتروني،

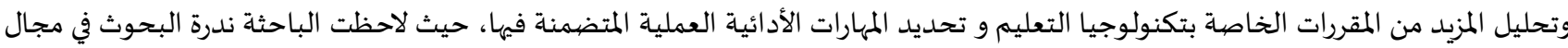

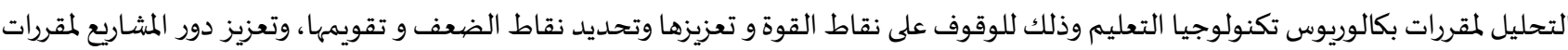
المواد العملية و ذلك ليستطيع الطالب بناء محتوى تعليمي الكتروني متكامل.

الكلمات المفتاحية : المهارات الأد ائية؛ مقررات؛ التصميم الجرافيكي للوسائط المرئية؛ Adobe Photoshop ؛ تكنولوجيا التعليم () (1)

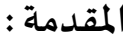

ظهر مصطلح تكنولوجيا التعليم حديثا نتيجة الثورة العلمية والتكنولوجية التي بدأت عام • ب ج ، عندما قام العالم جيمس فن (James Finn) رئيس قسم التعليم السمعي البصري في أمريكا بتأسيس لجنة لتعريف بعض المصطلحات المتعلقة بهذا المجال، والتي توصلت إلى أن مصطلح الوسائل التعليمية أصبح محدوداً ولا يستطيع أن يصف هذا المجال بدقة، وقد تطور مفهوم تقنيات التعليم في العالم العربي كي يقابل مصطلح تكنولوجيا

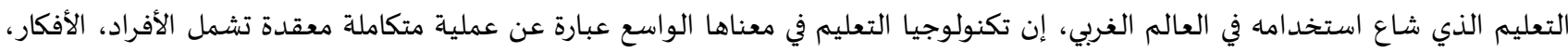

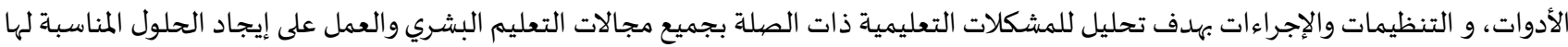

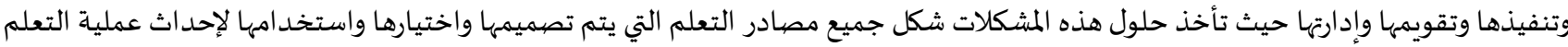
وتشتمل هذه المصادر على وسائل وأفراد ومواد وأدوات وأساليب ومراكز مصادر تعلم تأخذ على عاتقها مسؤولية تحديد عمليات تحليل المشكلات التعليمية وإيجاد الحلول المناسبة لها وتنفيذها وتقويمها ضيمن إطار وظائف التطوير التربوي المتعلقة ببحث النظرية، والتصميم والإنتاج والتقويم

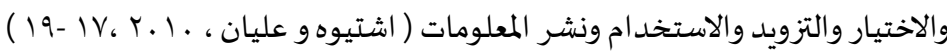
ويعد التعليم الجامعي المحرك الأسـاس في عملية التنمية بجميع أبعادها الإقتصادية والاجتماعية والثقافية، وحيث إن الجامعة مؤسسـة تقدم

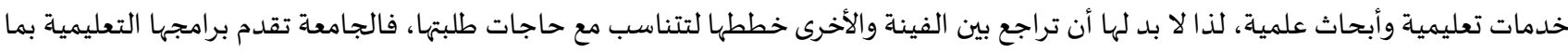
يتفق مع رغبات الطلبة وتوجهاتهم المستقبلية، والتي هي بالضرورة استطلاعات للطلب المتوقع على العلوم التي يدرسونها ـ ( الرحاحلة، 7 . . ب ) .

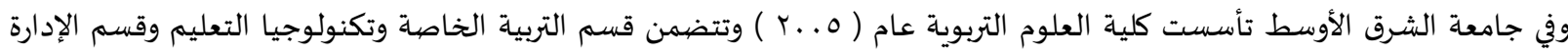

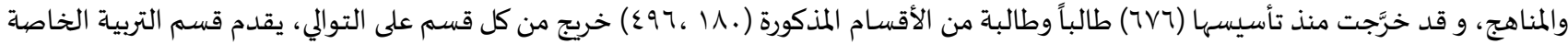

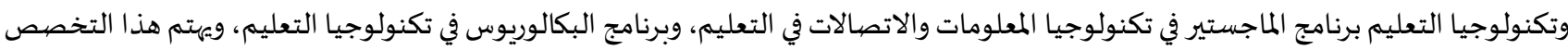


في استخدام و توظيف التكنولوجيا في عمليات التعلم والتعليم، وتصميم وإنتاج المواد التعليمية ودمجها في المناهج المختلفة، وتشجيع بيئة تعليمية تواكب الثورة المعرفية والحضارية السائدة التي يتم فيها التعليم باستخدام الحاسوب والوسائط المتعددة، والنظم الذكية في تصيميم وإدارة بيئات

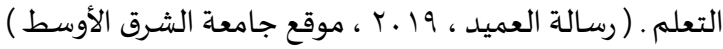

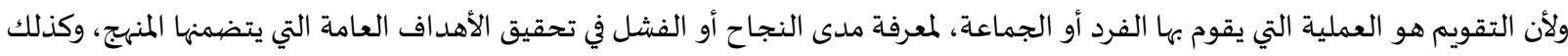

لمعرفة نقاط القوة والضعف باه حتى يمكن تحقيق الأهداف المنشودة بأحسن صورة.( الوكيل المفتي، ـ 199 ) لذلك كان لا بد من الحاجة إلى تقويم مقررات تكنولوجيا التعليم بما تحتويها من المهارات الأدائية الحركية، و تعني عملية التقويم كما عرَّفها

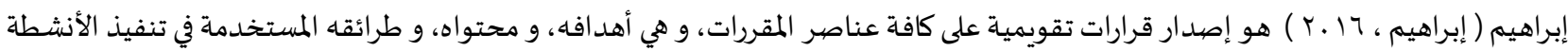
أما المهارات أو الأداءات العملية التي تستهدفها عملية التقويم للمقرر فهو الاسم الآخر الذي يطلق على المهارات النفسحركية،حيث تعرف

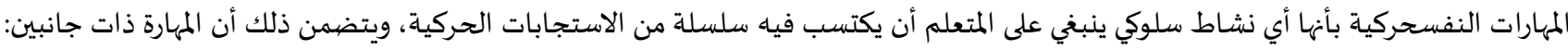
الجانب الأول نفسي، وفيه يدرك الفرد الحركة، ثم يفكر فهها ثم يستوعبها، والجانب الثاني يتمثل في ممارستها، ويمكن أن ينظر إلى البعد الحركي على أنها

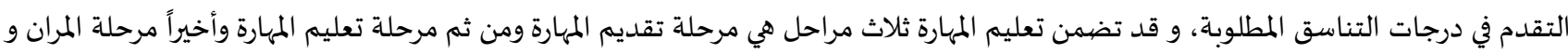
التدريب على المهارة، ويجري قياس وتقويم أي من الأهداف الأدائية ذات الصلة بالمهارات النفسحركية عن طريق ملاحظة الأداء وموازنتا بمعايير دقيقة

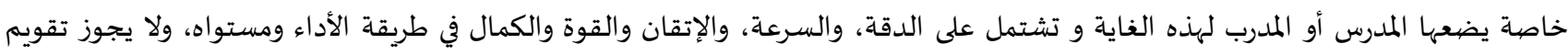

الأهد اف الأدائية في هذا المجال عن طريق الاختبارات الكتابية و الإنشائية أو الموضوعية إلا إذا كانت مهارة الكتابة هي الهدف. ( قطامي، م . . ب ) ويُعد مقرر التصميم الجرافيكي للوسائط المرئية في تخصص تكنولوجيا التعليم لدرجة البكالوريوس في جامعة الشرق الأوسط من المقررات

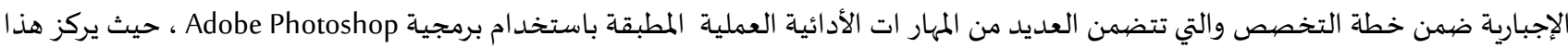
المساق على الجانب النظري و العملي في التصهيم الجرافيكي والنصوص في إنتاج مواد تعليمية مرئية من خلال استخدام برامج الحاسوب المختلفة في

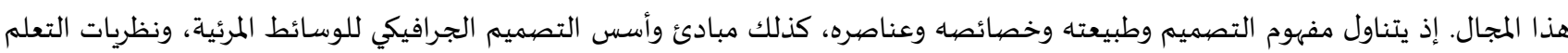
والاتصال المتعلقة بتصميم وإنتاج المواد التعليمية المستخدمة في التدريس والتدريب باستخدام البرمجيات والتقنيات الحديثة للحاسوب. (خطط

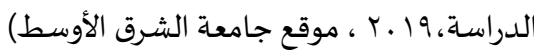

ويُعد برنامج أدوبي فوتوشوب Adobe Photoshop المستخدم في تطبيق المهارات الأدائية العملية في مقرر التصيميم الجرافيكي للوسائط المرئية

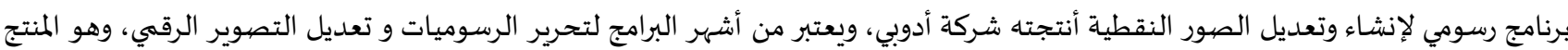

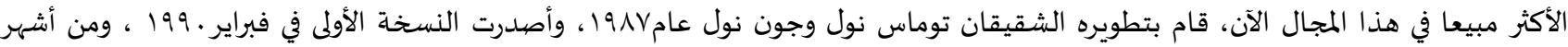
استخداماته إنشاء التصاميم البصرية المتنوعة، وذلك من خلال دمج الصور والنصوص وغيرها من العناصر مع بعضها البعض في تصهميم واحد، وتعديل الصيور الفوتوغرافية من خلال تغيير الألوان أو الإضياءة أو التباين، والتلاعب بالصهور والتصهميمات بإدخال أو إزالة أجزاء، كما يمكن تصميميم

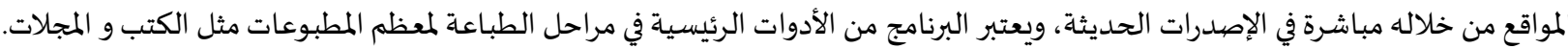

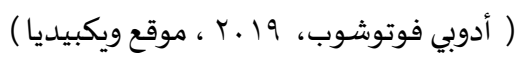

الدراسات السابقة:

وقد تناولت الباحثة عددًا من الدراسات السابقة العربية و الأجنبية ذات الصلة و منها: دراسة الحياري (VI إ) التي هدفت إلى تقويم مقررات تخصص تكنولوجيا التعليم لمرحلة البكالوريوس في الجامعات الأردنية الخاصة من وجهة

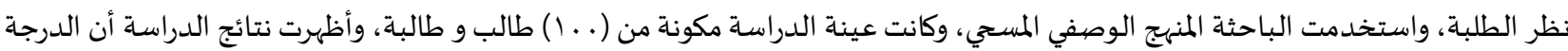
الكلية لوجهة نظر طلبة تخصص تكنولوجيا التعليم نحو مقررات التخصص كانت متوسطة، كما وأظهرت النتائج عدم وجود فروق ذات دلالة

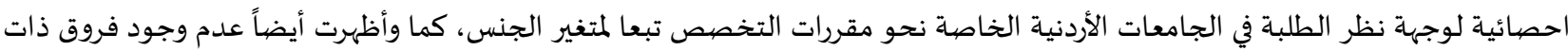
دلالة احصائية في مجالات: أهداف مقررات تكنولوجيا التعليم، وفاعلية مقررات تكنولوجيا التعليم، ومحتوى مقررات تكنولوجيا التعليم تبعاً لمتنير

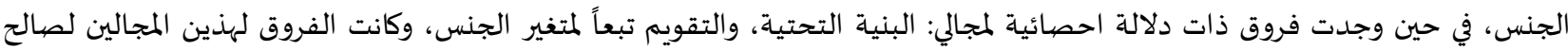

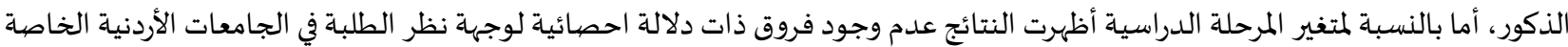
نحو مقررات التخصص، تبعاً لمتغير المرحلة الدراسية . وهدفت دراسة إبراهيم (17 • إلى تقويم مقرر التصميم التعليمي لماجستير تكنولوجيا التعليم في كلية التربية في جامعة السودان للعلوم والتكنولوجيا، في ضوء معايير الجودة الشاملة، وذلك من خلال تحديد أهداف ومحتوى مقرر التصميم التعليمي لمعايير الجودة، بالإضافة إلى

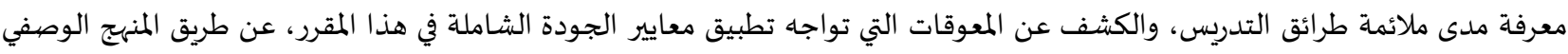

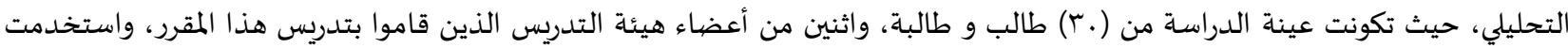


الباحثة الإستبانة والمقابلة و قائمة تحليل المحتوى لجمع البيانات، وقد توصلت الدراسة إلى أن مقرر التصهيم التعليمي لماجستير تكنولوجيا

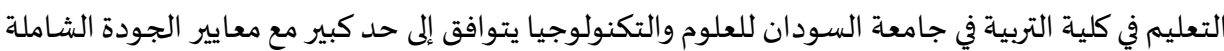
وهدفت دراسـة عبد العليم (1) ـ إلى تقصي أثر التفاعل بين أنماط مساعدات التعلم ومستويات تقديمها ببيئات التعلم المصغر عبر الويب الجوال في تنمياة مهارات البرمجة و القابلية للاستخدام لدى طلاب تكنولوجيا التعليم، وذلك من خلال اعداد قائمة بمهارات البرمجة بلغة Basic.Net المتضمنة في مقرر البرمجة لطلاب الفرقة الثانية قسم تكنولوجيا التعليم، وتحديد معايير تقديم مساعدات التعلم ببيئات التهات التعلم

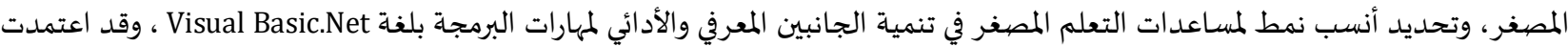

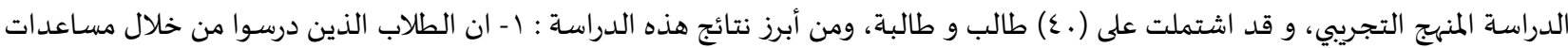
التعلم عند الطلب كانوا أكثر إيجابية في تحصيل الجانب المعرفي للمهارات مقارنة مع الطلاب الذين درسوا من خلال المساعدات المستمرة . r -

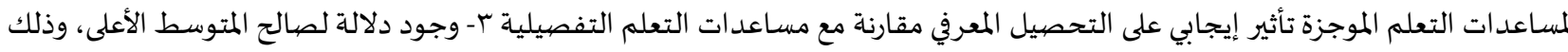
للمجموعاة التجريبية التي استخدمت مساعدات التعلم عند الطلب. و هدفت دراسـة شتات (7 . . Y) لتقويم مقررات الحاسوب المتقدمة لطلبة كلية العلوم التربوية من مستوى البكالوريوس في الجامعة الأردنية في

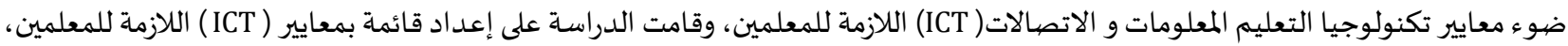
بالإضافة إلى وضع تصور لمقررات الحاسوب لطلبة كلية العلوم التربوية من مستوى البكالوريوس في الجامعة الأردنية لمعايير ( ICT) اللازمة للمعلمين. كما و هدفت دراسـة أبو الحاج (9 . . r) إلى تقويم برنامج الدبلوم العالي في تكنولوجيا المعلومات و الاتصالات في التربية ( ICTE) في الجامعة الأردنية

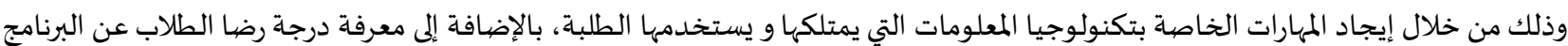
وأبرز الصعوبات التي تواجه الخريجين في استخدام المهارات الخاصة بتكنولوجيا المعلومات والاتصالات في التربية في ممارساتهم التعليمية، وقد

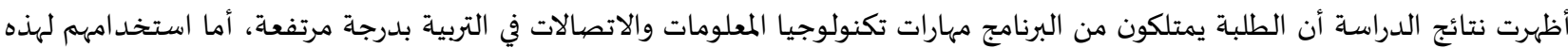

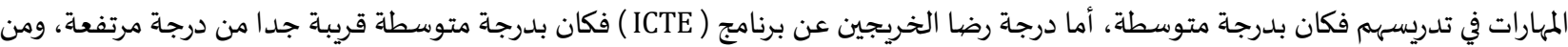

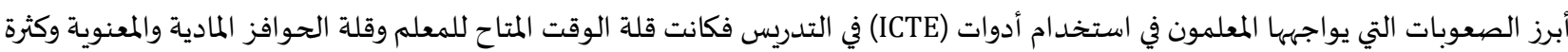
اعداد الطلبة وقلة البرامج التعليمية المتوفرة في المدرسة. وهدفت دراسـة العفتان (9 . . ا إلى دراسة درجة استخدام طلبة الجامعة العربية المفتوحة للتعلم الإلكتروني من وجهة نظر أعضاء هيئة التدريس والطلبة في الجامعة، وهل تختلف درجة استخدام طلبة الجامعة العربية المفتوحة للتعلم الإلكتروني باختلاف تقديرات كل من أعضياء هيئة

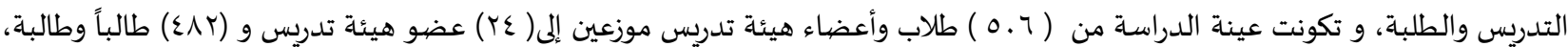

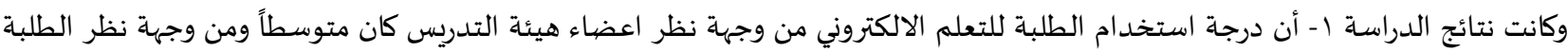

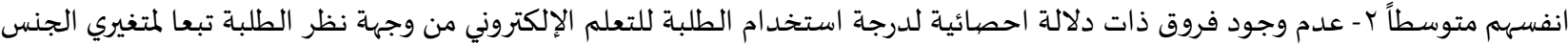

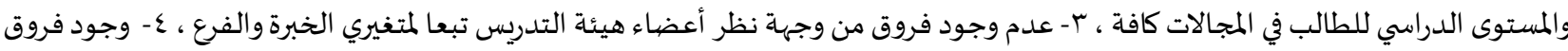

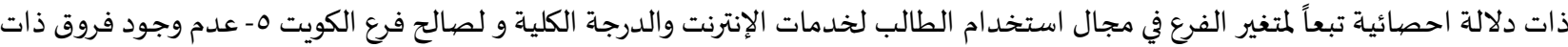

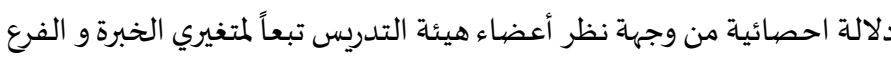

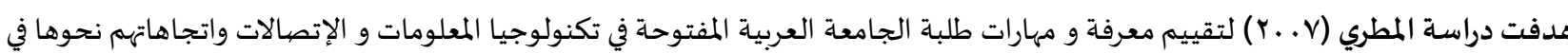

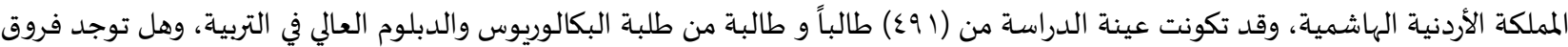

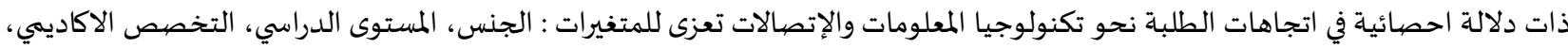
السلطة المشرفة على شهادة الثانوية العامة، وقد أظهرت نتائج الدراسة ا - أن برمجية معالجة النصوص قد احتل المرتبة الأولى في التقييم الذاتي

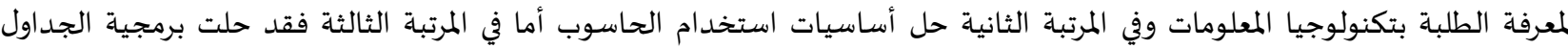

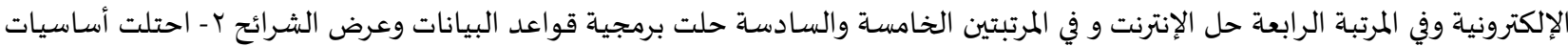

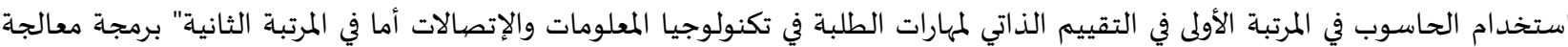

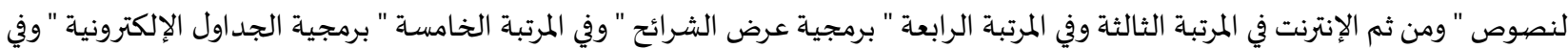
المرتبة الأخيرة " برمجية قواعد البيانات

وهدفت دراسة العوهلي (Y . . إ) إلى تقويم برنامج وسائل وتكنولوجيا التعليم لدرجة الماجستير في جامعة الملك سعود بالمملكة العربية السعودية من وجهة نظر أعضاء هيئة التدريس والطلبة، و إيجاد إذا كان هناك فروق ذات دلالة احصائية في تقديرات أفراد عينة الدراسة لواقع برنامج

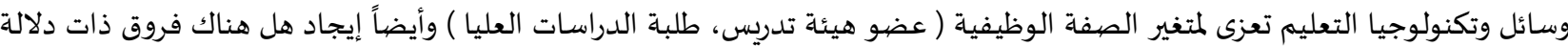

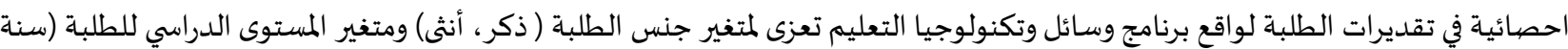


أولى، سنة ثانية، سنة ثالثة)، و قد شملت عينة الدراسة( V ) من أعضاء هيئة التدريس و ( T ) طالباً و طالبة تم اختيارها بالطريقة العشوائية ..

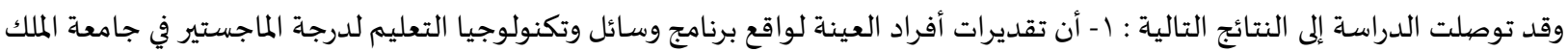
سعود في المملكة العربية السعودية كانت كبيرة من وجهة نظر أعضاء هيئة التدريس في حين كانت بدرجة متوسطة من وجهة نظر طلبة الدراسات

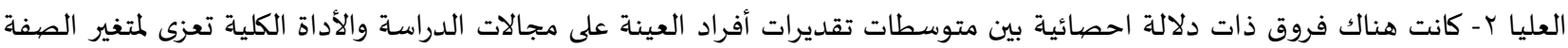

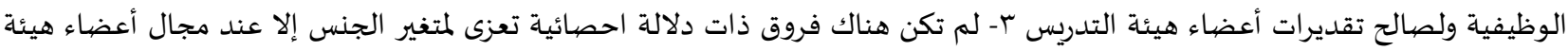
التدريس ومجال أساليب تقويم البرنامج و لصالح الذكور ع - لم تكن هناك فروق ذات دلالة احصيائية تعزى لمتغير المستوى الدراسي . هدفت دراسة (Anison, 2002) إلى تقييم برنامج الدبلوم العالي للتعلم عن بعد في تكنولوجيا المعلومات والإتصالات في جامعة بلايموث البريطانية،

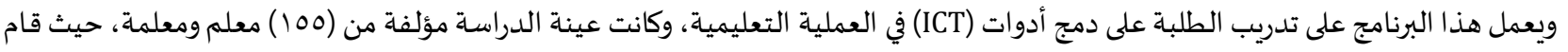

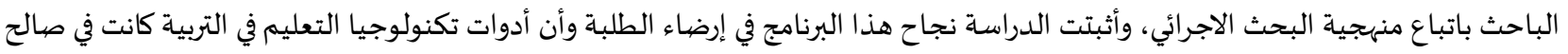

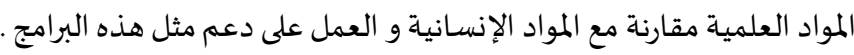
و هدفت دراسة كورزما (Kozma, 2004) إلى تقويم برنامج World link في الدول النامية و الدول المتطورة ومستوى الخدمات التي يقدمها البرنامج، وأظهرت الدراسة أن البرنامج قدم الأجهزة والبرمجيات التي تساعد المتعلمين على تعلم أفضل .

مشكلة الدراسة و أسئلتها: جاءت هذه الدراسة للبحث وتحديد المهارات الأدائية (العملية) في مقرر التصيميم الجرافيكي للوسائط المرئية في تخصص تكنولوجيا التعليم لدرجة البكالوريوس في جامعة الشرق الأوسط، وتحديد مدى تطبيق الطلبة للمهارات الأدائية (العملية) التي يتضمنها المقرر والمطبق باستخدام برنامج من وجهة نظرهم، وذلك لتطوير العملية التربوية وتحديد مواطن القوة لتعزيزها و تحديد مواطن الضعف لتصدية (Adobe Photoshop cc) على توصيات بعض الدراسات السـابقة التي أوصت بضرورة وجود دراسات تتناول تقويم مقررات تخصص تكنولوجيا التعليم مثل العوهلي (r ... (Y)،

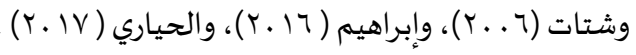

أسئلة الدراسـة: ا. ما المهارات الأدائية (العملية) التي يتضمنها مقرر التصميم الجرافيكي للوسائط المرئية في تخصص تكنولوجيا التعليم لدرجة البكالوريوس في

$$
\text { جامعة الشرق الأوسط. }
$$
r. ما مدى تطبيق طلبة بكالوريوس تكنولوجيا التعليم في جامعة الشرق الأوسط للمهارات الأدائية (العملية) التي يتضمنها مقرر التصميم الجرافيكي للوسائط المرئية من وجهة نظرهم

أهمية الدراسـة: ا. من المؤمل أن توفر هذه الدراسة لصانعي القرار معلومات عن المهارات الأدائية (العملية) التي يوفرها مقرر التصميم الجرافيكي للوسائط المرئية في تخصص تكنولوجيا التعليم لدرجة البكالوريوس في جامعة الشرق الأوسط، ومدى تطبيق الطلبة لهذه المهارات في تصهيم وسائط مرئية ومحتوى تعليمي تكنولوجي من وجهة نظرهم.

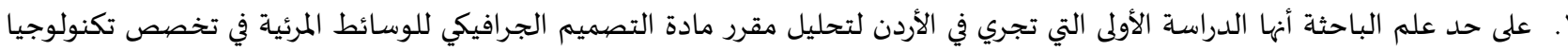

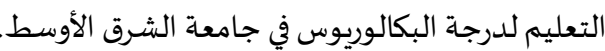

r. تفتح المجال أمام الباحثين لدراسة و تحليل مقررات أخرى لتخصص تكنولوجيا التعليم لدرجة البكالوريوس في جامعة الشرق الأوسط. ع. يتوقع أن تسهم في إعادة النظر في طرح مواد تتضمن مهارات أدائية عملية لدعم قدرة الطلبة على تصميم محتوى تعليمي إلكتروني متقدم.

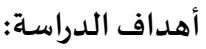

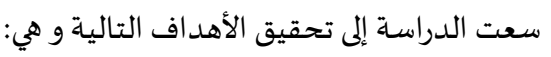
ا. تحديد المهارات الأدائية (العملية) التي يتضمنها مقرر التصميم الجرافيكي للوسائط المرئية في تخصص تكنولوجيا التعليم لدرجة البكالوريوس في جامعة الشرق الأوسط. r. التعرف إلى مدى تطبيق طلبة بكالوريوس تكنولوجيا التعليم في جامعة الشرق الأوسط للمهارات الأدائية (العملية) التي يتضمنها مقرر التصميم الجرافيكي للوسائط المرئية من وجهاية نظرهم. حدود الدراسـة: الحدود المكانية: جامعة الشرق الأوسط 


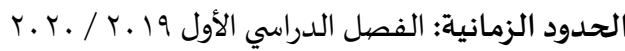
الحدود البشرية: طلبة تخصص تكنولوجيا التعليم.

الحدود الموضوعية: استخدمت أداة تحليل المحتوى في استخراج المهارات الأدائية العملية التي يتضمنها مقرر التصميم الجرافيكي للوسائط المرئية

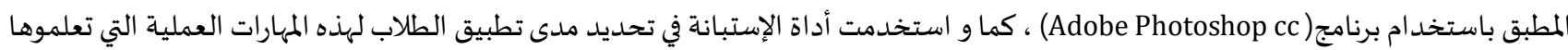

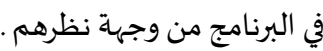

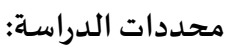

صيدق أداة الدراسة و ثباتها .

صدق و جدية أفراد العينة عند الإجابة عن فقرات الإستبانة .

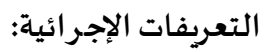

تخصص تكنولوجيا التعليم: وهو أحد البرامج الدراسية في جامعة الشرق الأوسط، و مدة الدراسة فيه أربع سنوات دراسية بواقع ( r آI )

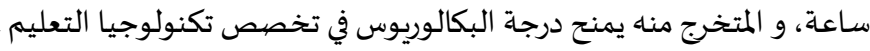
طلبة تخصص تكنولوجيا التعليم: وهم عينة من طلبة بكالوريوس تكنولوجيا التعليم في جامعة الشرق الأوسط للفصل الدراسي الأول للعام

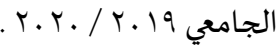

مقررالتصميم الجرافيكي للوسائط المرئية: وهو أحد مقررات تخصص بكالوريوس تكنولوجيا التعليم في جامعة الشرق الأوسط، حيث يركز

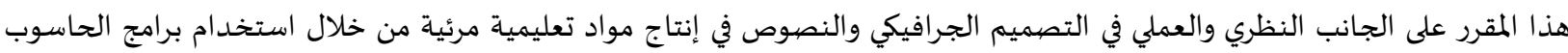
المختلفة في هذا المجال. إذ يتناول مفهوم التصيميم وطبيعته وخصيائصها وعناصره، كذلك مبادئ وأسس التصيميم الجرافيكي للوسائط المرئية، ونظريات التعلم والإتصال المتعلقة بتصيميم وإنتاج المواد التعليمية المستخدمة في التدريس والتدريب باستخدام البرمجيات والتقنيات الحديثة للحاسوب باستخدام برنامج (Adobe Photoshop cc) برنامج(Adobe Photoshop cc) : هو برنامج رسوم لإنشاء وتعديل الصور النقطية أنتجته شركة أدوبي ويعتبر أشهر البرامج لتحرير الرسوميات وتعديل التصيوير الرقمي. المهارات الأدائية (العملية): وهي المهارات التطبيقية العملية لبرنامج (Adobe Photoshop cc) المتضيمنة والمطبقة في مقرر مادة التصميم

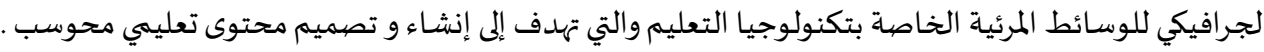

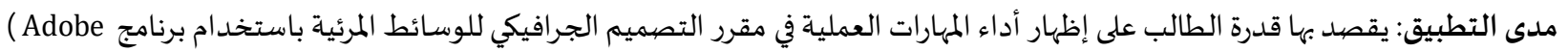
Photoshop cc)

إجراءات الدراسـة:

بعد اعتماد أداة الدراسة بصورتها الأولية، و لتحقيق أهداف الدراسة اعتمدت الباحثة الخطوات الآتية:

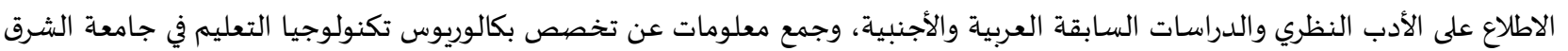
الأوسط وذلك بالاطلاع على خطة وصف مواده ومحتوى هذه المواد. قامت الباحثة باختيار مقرر التصميم الجرافيكي للوسائط المرئية والمطبق باستخدام برمجية Adobe Photoshop cc

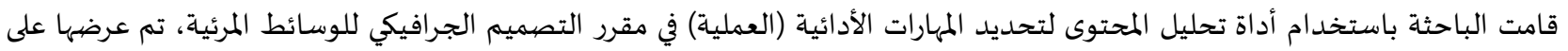

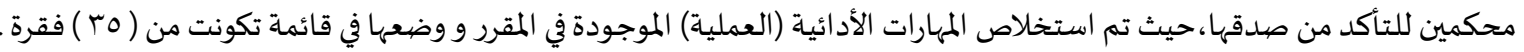
تحديد مجتمع الدراسة والعينة. تم بناء أداة الاستبانة بناء على أداة تحليل المحتوى بحيث تضيمنت جميع البنود الموجودة في أداة تحليل المحتوى وتم اختبار ثباتها عن طريق ألفا كرونباخ للثبات الداخلي. تم توزيع وتطبيق الاستبانة على أفراد عينة الدراسة ورصد البيانات في جداول خاصية، وإجراء معالجة احصائية مناسبة للاستبانة باستخدام الرزمة الحصائية (SPSS) واستخراج النتائج. قامت الباحثة بمناقشة النتائج وتقديم التوصيات بناء على نتائج الدراسة. 
اعتمدت الباحثة في دراستها الميدانية المنهج الوصفي بشقيه النوعي والكمي، وذلك لما لاتباع هذا المنهج من قدرة في توفير كمية من البيانات

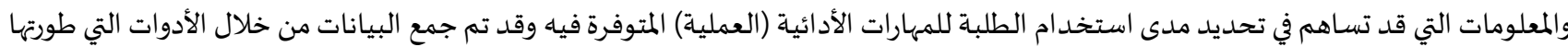
الباحثة وهي عبارة عن أداتين: الأولى: أداة تحليل المحتوى وهي أداة بحث نوهي ادهي تم استخدامها لاستيتخلاص المهارات الأدائية العملية الموجودة في مقرر التصيميم الجرافيكي للوسائط المرئية المطبق باستخدام برنامج (الادئ (Adobe Photoshop cc)

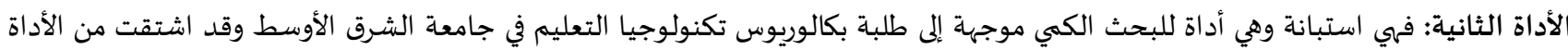
الأولى، وقد تكونت الإستبانة من جزأين الأول وهو عبارة عن معلومات ديموغرافية عن الطلباة، والجزء الثاني رأي الطلبة في مدى تطبيقهم للمهارات

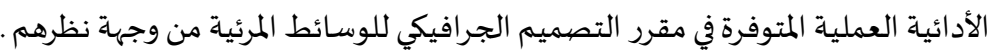

الطريقة و الإجراءات: مجتمع الدراسـة:

تألف مجتمع الدراسة الأول من جميع المواد الموجودة في تخصص بكالوريوس تكنولوجيا التعليم في جامعة الشرق الأوسط ويبلغ عددها (بrا ( )

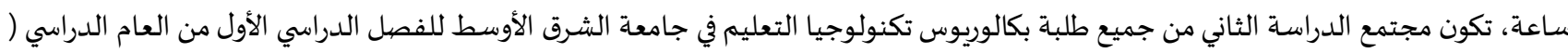
( r. r. - r. 19 عينة الدراسـة:

تكونت عينة الدراسـةالأولى من مقرر التصميم الجرافيكي للوسائط المرئية المطبق باستخدام برنامج( (Adobe Photoshop cc) ، المطروحة ضمن

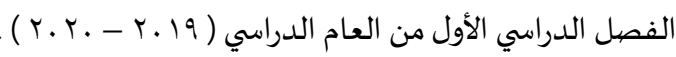

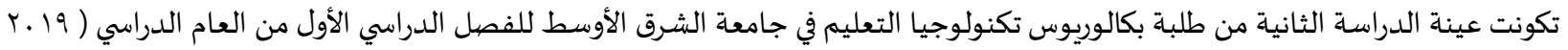

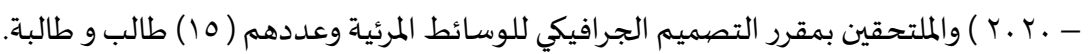
أدوات الدراسـة:

أداة تحليل المحتوى: وقد احتوت على قائمة بالمهارات الأدائية (العملية) الخاصة باستخدام تكنولوجيا التعليم الموجودة في مقرر التصميم

الجرافيكي للوسائط المرئية المطبقة باستخدام برنامج(Adobe Photoshop cc) و قد تضيمنت ( (T) مهارة أد ائية ( عملية ) .

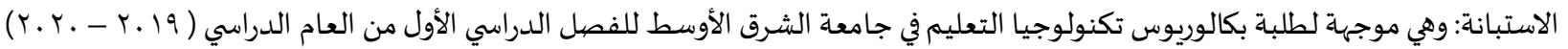
و الملتحقين بمقرر التصميم الجرافيكي للوسائط المرئية، وتم تطويرها من قبل الباحثة بحيث تكوجيا تكونت من جن جزأين:

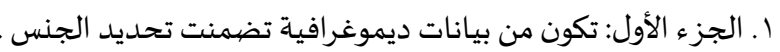

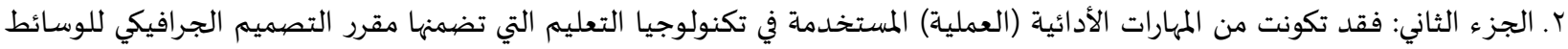
المرئية لتخصص بكالوريوس تكنولوجيا التعليم ومدى تطبيق الطلاب لها من وجهة نظرهم، وقد تضيمنت ( مجاء ) مهارة أدائية ( عملية) ، وقد تم

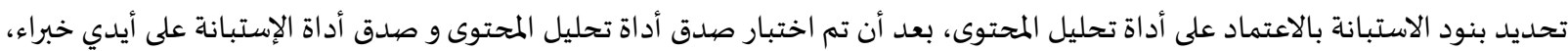

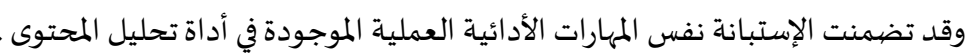

صيدق أدوات الدراسـة:

قامت الباحثة لأجل التحقق من صدق أدوات الدراسة بعرضها على محكمين من أعضاء هيئة التدريس لتخصص بكالوريوس تكنولوجيا التعليم في جامعة الشرق الأوسط فكانت أن أعطيت موافقة على تحليل المحتوى لمقرر التصميم الجرافيكي للوسائط المرئية من قبل المحكمين مع ابداء بعض الماحظات التي أخذت بعين الاعتبار و صححت على أساسـاه وتم ارجاعها للمحكمين للإطلاع على التعديلات وتم الحصول على على الموافقة النهائية

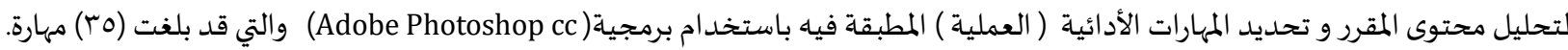

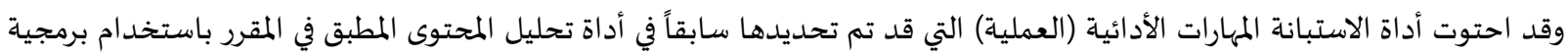
وقد تم اختبار صدقها من خلال عرضها على المحكمين من أعضاء هيئة التدريس لتخصص بكالوريوس تكنولوجيا التعليم في جامعة الشرق الأوسط، وقد ظهرت الاستبانة بصورتها النهائية وقد احتوت على (ب0) مهارة عملية لقياس مدى تطبيق الطلاب لهذه المهارات من وجهاة نظرهم. 
جدول ( ) : نتائج ثبات المهارات الأدائية ( العملية ) باستخدام طريقة ألفا كرونباخ للثبات الداخلي

\begin{tabular}{|c|c|c|}
\hline كرونباخ ألفا & عدد الفقرات & المهارات الأدائية ( العملية ) \\
\hline $.9 \mathrm{VV}$ & ro & التصيميم الجرافيكي للوسائط المرئية \\
\hline
\end{tabular}

يبين الجدول (1) نتائج ثبات المهارات الأدائية (العملية) باستخدام طريقة ألفا كرونباخ للثبات الداخلي، و باستعراض قيم ألفا كرونباخ

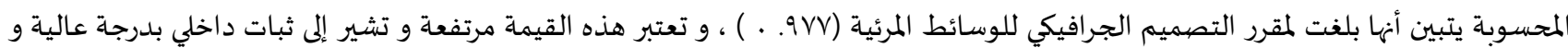

المعالجة الاحصيائية: مقبوله

قامت الباحثة باستخدام المعالجات الاحصائية المتوافرة ضيمن حزمة برامج (SPSS) وبالتحديد استخدمت المتوسطات الحسـابية - الانحرافات

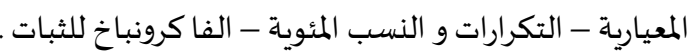

$$
\text { نتائج الدراسة و مناقشتها : }
$$

للاجابة عن السؤال الأول ونصه: ما المهارات الأدائية (العملية) التي يتضمنها مقرر التصميم الجرافيكي للوسائط المرئية في تخصص تكنولوجيا

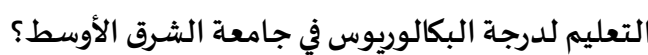

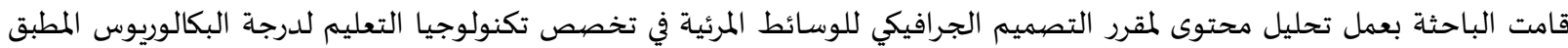

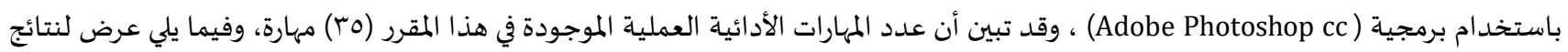
التحليل لمقرر التصيميم الجرافيكي للوسائط المرئية .

جدول(ץ): المهارات الأدائية ( العملية) التي يتضهنها مقرر التصهيم الجرافيكي للوسائط المرئية

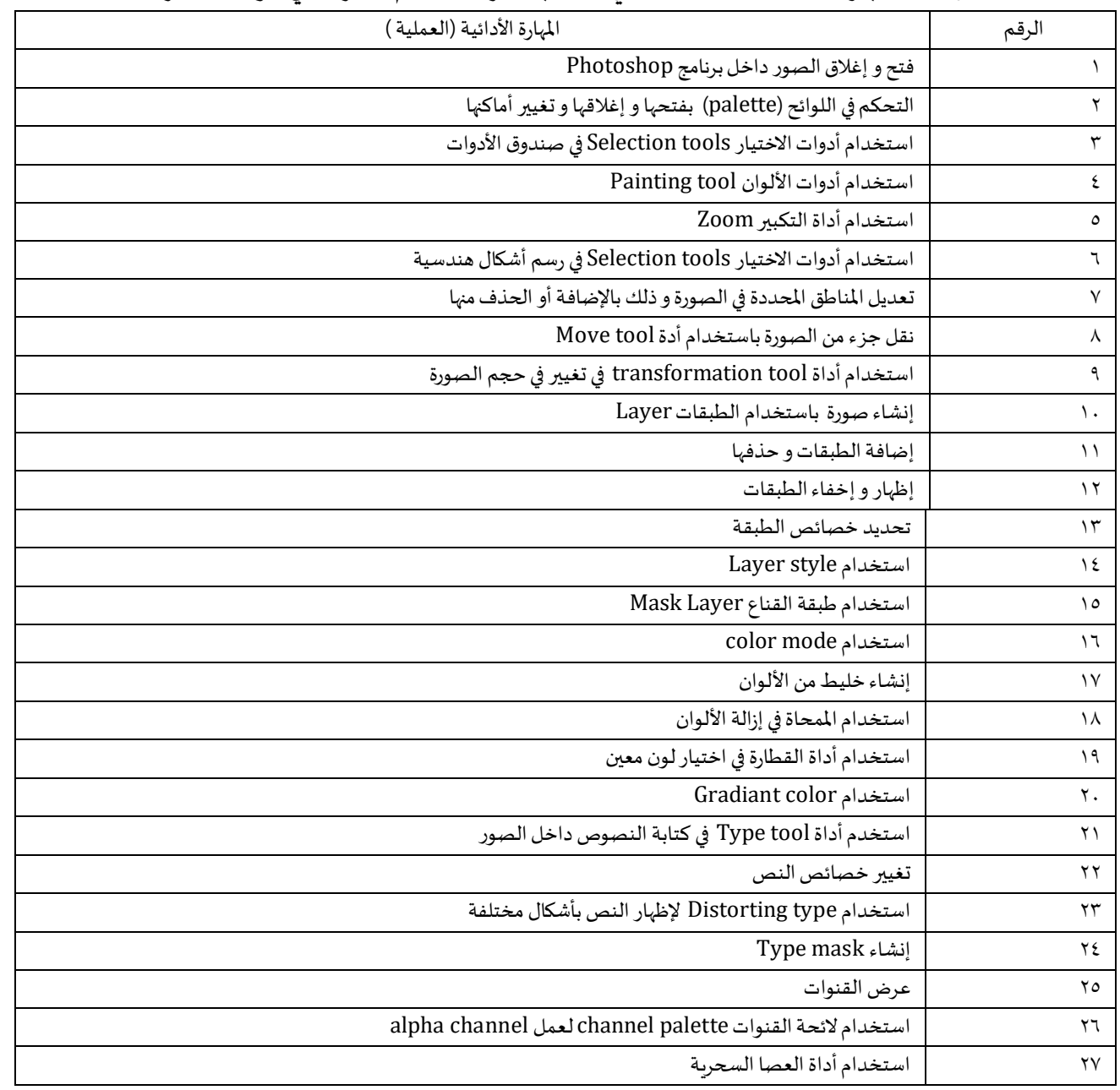




\begin{tabular}{|c|c|c|}
\hline & Filter gallery استخدام & ru \\
\hline & استخدام أكثر من فلتر و قناع مع نفس الصهورة & rq \\
\hline & استخدام Displacement لإضافة العمق للصورة & $r$. \\
\hline & Pattern إنشاء و استخدام & ri \\
\hline & استخدام Pen tool لعمل الأشكال Vector & rT \\
\hline & عمل optimization للصور لتقليل حجمها & Tr \\
\hline & حفظ الصورة لتكون صالحة للاستخدام في الويب & $r \varepsilon$ \\
\hline & عمل خريطة صورية image map & ro \\
\hline
\end{tabular}

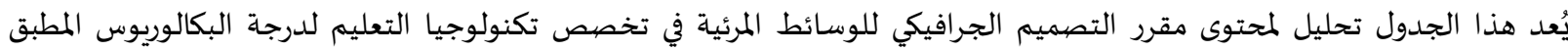
باستخدام برمجية (Adobe Photoshop cc) ) ، و قد تبين أن عدد المهارات الأدائية العملية الموجودة في هذا لمدائية المقرر (ro) مهارة . النتائج المتعلقة بالسؤال الثاني ومناقشتهـ:

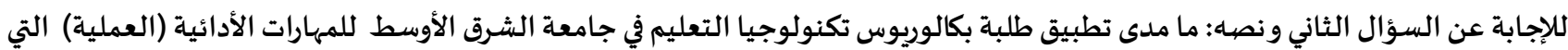

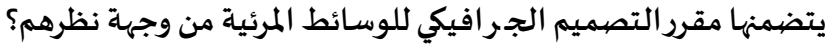

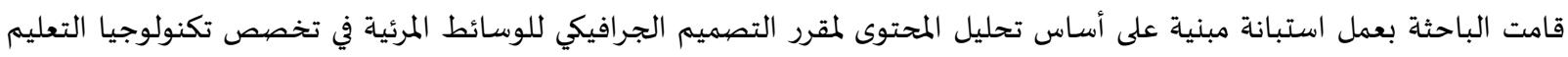
لدرجة البكالوريوس المطبق باستخدام برمجية (Adobe Photoshop cc) ، وتوزيعها على الطلبة ومن ثم القيام بتحليلها احصائيا للحصول على النتائج والتي كانت كالتالي: تم حساب المتوسطات الحسابية والانحرافات المعيارية والنسب المئوية و التصنيف لمدى تطبيق طلبة بكالوريوس تكنولوجيا التعليم في جامعة

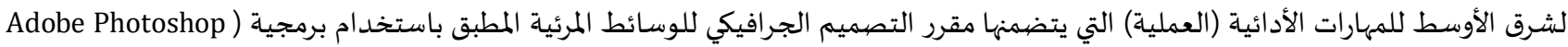

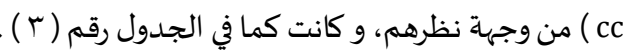

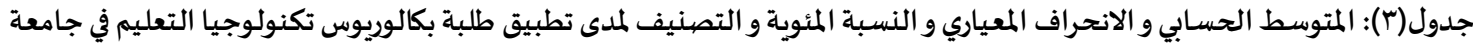

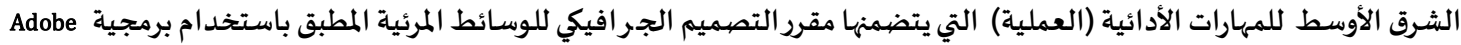

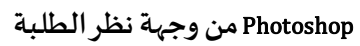

\begin{tabular}{|c|c|c|c|c|}
\hline التصنيف & النسبة المئوية & الانحراف المعياري & المتوسط & المقرر \\
\hline عالية & v9,71 &., $\mathrm{v}$. & $r, 91$ & التصميم الجرافيكي للوسائط \\
\hline
\end{tabular}

باستعراض قيمة النسبة المئوية نجد أن مدى تطبيق طلبة بكالوريوس تكنولوجيا التعليم في جامعة الشرق الأوسط للمهارات الأدائية ( العملية

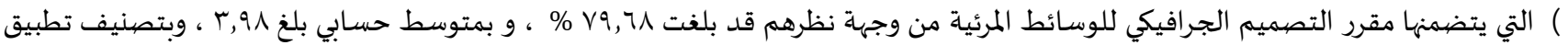

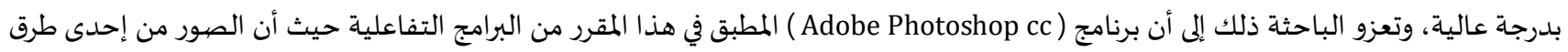

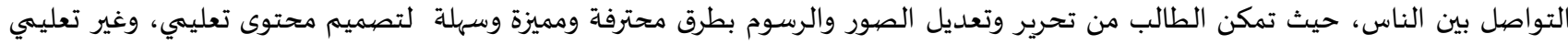

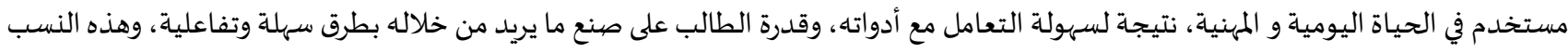

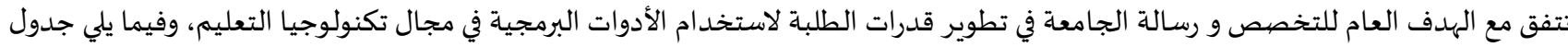

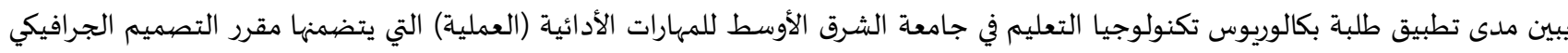

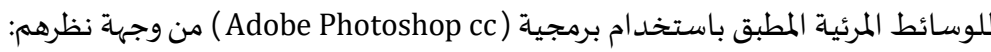

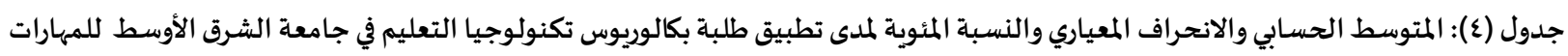

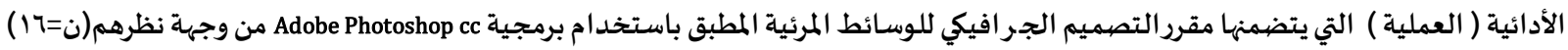

\begin{tabular}{|c|c|c|c|c|c|}
\hline التصنيف & الترتيب & المئوية & الانحراف & المتوسط & 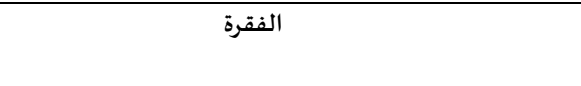 \\
\hline 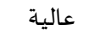 & 1 & 91, ro & $\cdot, \mathrm{V} r$ & $\varepsilon, 07$ & فتح و إغلاق الصور داخل برنامج Photoshop \\
\hline عالية الية & r & M, vo & $\cdot, \mathrm{Vr}$ & $\varepsilon, \varepsilon \varepsilon$ & التحكم في اللوائح (palette) بفتحهاو إغلاقها و تغيير أماكنها \\
\hline عالية & 7 & ᄉ7, ro &., $1 \vee 9$ & $\varepsilon, r_{1}$ & استخدام أدوات الإختيار Selection tools في صندوق الأدوات \\
\hline عالية & r & M , vo &., $\mathrm{VT}$ & $\varepsilon, \varepsilon \varepsilon$ & Painting tool استخدام أدوات الألوان \\
\hline 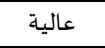 & $\varepsilon$ & $\wedge \mathrm{V}, 0$ &., $\mathrm{VT}$ & $\varepsilon, \Upsilon \wedge$ & استخدام أداة التكبير Zoom استخبر \\
\hline عالية & 0 & $\wedge \mathrm{V}, 0$. &., $\mathrm{VT}$ & $\varepsilon$, « & استخدام أدوات الإختيار Selection tools في رسم أشكال هندسية \\
\hline
\end{tabular}




\begin{tabular}{|c|c|c|c|c|c|}
\hline عالية & v & $10, \ldots$ &., $\mathrm{VV}$ & $\varepsilon$, YO $_{0}$ & تعديل المناطق المحددة في الصهورة و ذلك بالإضافة أو الحذف منها \\
\hline عالية & 1. & $\Delta r, v_{0}$ &., 91 & $\varepsilon, 19$ & نقل جزء من الصورة باستخدام أداة Move tool \\
\hline عالية & 10 & $11, r_{0}$ &., $\mathrm{VV}$ & $\varepsilon, .7$ & استخدام أداة transformation tool في تغيير حجم الصورة \\
\hline عالية & ri & $v_{\wedge}, v_{0}$ &., 94 & $r, q \varepsilon$ & إنشاء صورة باستخدام الطبقات Layer \\
\hline عالية & 11 & $\Lambda_{.}, \ldots$ & $\cdot, \wedge 9$ & $\varepsilon, .$. & إضافة الطبقات و حذفها \\
\hline عالية & 11 & $\Delta r, O$. &., 97 & $\varepsilon, 1 r$ & إظهار و إخفاء الطبقات \\
\hline عالية & 19 & $\Lambda_{1, \ldots}$ &., $9 \mathrm{~V}$ & $\varepsilon, .$. & تحديد خصائص الطبقة \\
\hline عالية & $r \mu$ & $V Y, 0$. & $1, . r$ & $r, \Lambda \Lambda$ & استخدام Layer style \\
\hline عالية & TV & V7, ro & $1, .0$ & $r, \wedge 1$ & استخدام طبقة القناع Mask Layer \\
\hline 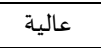 & 17 & $11, r_{0}$ & $\cdot, \mathrm{VV}$ & $\varepsilon, .7$ & استخدام color mode \\
\hline عالية & rr & $\mathrm{VA}, \mathrm{VO}$ &., 10 & r, १ะ & إنشاء خليط من الألوان \\
\hline 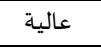 & $\wedge$ & $10, \ldots$ &., 94 & $\varepsilon, r_{0}$ & استخدام الممحاة في إزالة الألوان \\
\hline 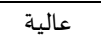 & r. & $\Lambda_{0}, \ldots$ & $1, \cdot r$ & $\varepsilon, .$. & استخدام أداة القطارة في اختيار لون معين \\
\hline عالية & iV & $11, r_{0}$ & $\cdot, 10$ & $\varepsilon, .7$ & Gradiant color Gتخدام \\
\hline عالية & IT & $\Delta T, O$. & $\cdot, \wedge 9$ & $\varepsilon, 1 r$ & استخدم أداة Type tool في كتابة النصوص داخل الصور \\
\hline عالية & 9 & $10, \ldots$ & $\cdot, 71$ & $\varepsilon$, YO $_{0}$ & تغيير خصائص النص \\
\hline عالية & $r \varepsilon$ & $\mathrm{vr}, \mathrm{O}$. &., 97 & $r, \Lambda \Lambda$ & استخدام Distorting type لإظهار النص بأشكال مختلفة \\
\hline عالية & ru & $v_{0}, \ldots$ & $1, \ldots$ & r,vo & إنشاء Type mask \\
\hline متوسطة & rr & $T Y, 0$. & 1,10 & r, זی & عرض القنوات \\
\hline متوسطة & $r \varepsilon$ & $T Y, 0$. & $1, r 7$ & $r, r \Lambda$ & استخدام لائحة القنوات Calpha channel لعمل channel palette \\
\hline عالية & ir & $\Delta r, 0$. & $\cdot, 19$ & $\varepsilon, 1 r$ & استخدام أداة العصا السحرية magic tool \\
\hline عالية & ro & $\mathrm{V} V, 0$. & $\cdot, 19$ & $r, \Lambda \Lambda$ & Filter gallery استخدام \\
\hline متوسطة & $r$ & VI, ro & $1, r)$ & $r, 07$ & استخدام أكثر من فلتر و قناع مع نفس الصورة \\
\hline متوسطة & ro & $70, \ldots$ & $1, .7$ & r, ro & استخدام Displacement لإضافة العمق للصورة \\
\hline عالية & r7 & $V Y, 0$. & $\cdot, 19$ & $r, \Lambda \Lambda$ & إنشاءو استخدام Pattern \\
\hline عالية & rq & $V r^{\prime}, V_{0}$ & $1,1 \varepsilon$ & $r, 79$ & استخدام Pen tool لعمل الأشكال Vector \\
\hline متوسطة & $r$. & VT,O. & $1, .9$ & r, & عمل optimization للصهور لتقليل حجمها \\
\hline عالية ( مالية & $1 \varepsilon$ & $\Delta r, O$. &., 97 & $\varepsilon, 1 \Gamma$ & حفظ الصورة لتصبح صالحة للاستخدام في الويب \\
\hline متوسطة & rt & $v_{\cdot, \ldots}$ & $1,1$. & $r, o$. & عمل خريطة صورية image map \\
\hline عالية & & $v 9,7 \wedge$ & ., V. & $r, 91$ & مدالى تطبيق طلبة البكالوريوس للمهيارات العملية الموجودة في مادة التصميم \\
\hline
\end{tabular}

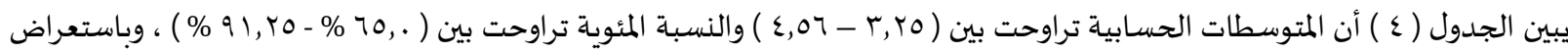

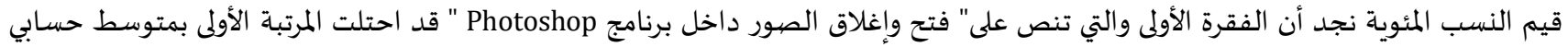

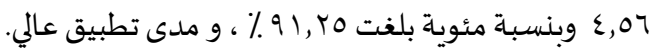
وفي المرتبة الثانية احتلت الفقرة الثانية " التحكم في اللوائح (palette) بفتحها و إغلاقها وتغيير أماكنها " بمتوسط حسابي (عـ,ع) وبنسبة

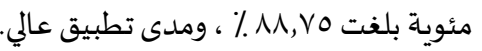
وتعزو الباحثة ذلك إلى أن برنامج ( Adobe Photoshop cc) هو برنامج لتحرير وتعديل الصهور وهذه من المهارات الأساسية والبسيطة والسهلة

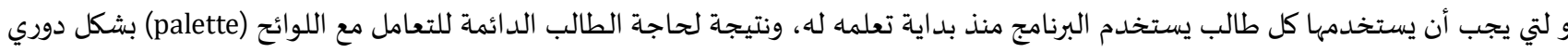

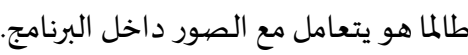

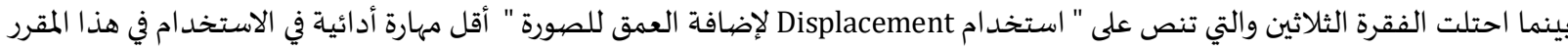

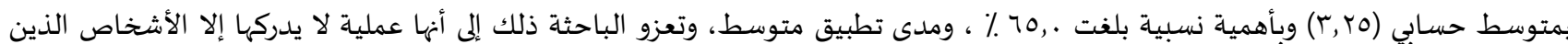
يمتلكون نظرة وبعد فني للصور و الرسومات.

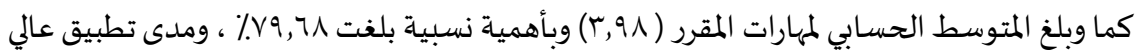


تحليل المزيد من المقررات الخاصة بتكنولوجيا التعليم وتحديد المهارات الأدائية العملية المتضمنة فيها، حيث لاحظت الباحثة ندرة البحوث في مجال التحليل لمقررات بكالوريوس تكنولوجيا التعليم وذلك للوقوف على نقاط القوة وتعزيزها وتحديد نقاط الضعف وتهبه وتقويمها . تعزيز دور المشاريع لمقررات المواد العملية في نهاية كل فصل وذلك ليستطيع الطالب بناء محتوى تعليمي إلكتروني متكامل باستخدام أدوات البرمجية التي تعلمها خلال المقرر . معردئ.

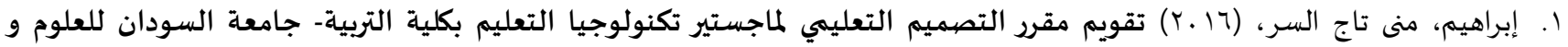

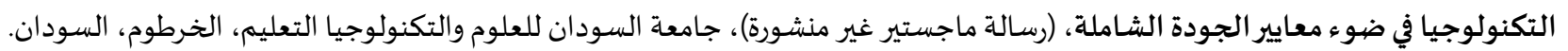

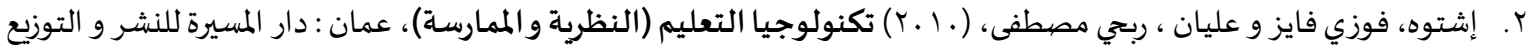

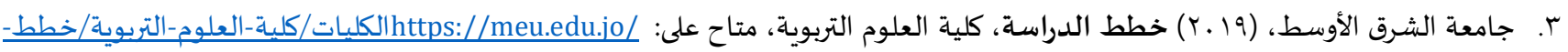
الدراسة

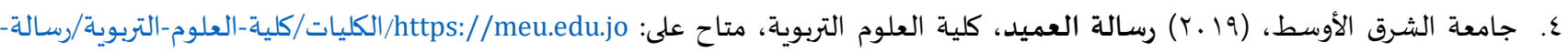
العميد

0. أبو الحاج، عطا أحمد حسن، (9 . . د) دراسة تقويمية لبرنامج الدبلوم العالي في تكنولوجيا المعلومات والإتصالات في التربية (ICTE) في الجامعة

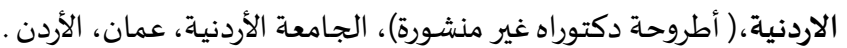

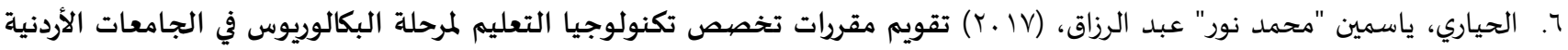

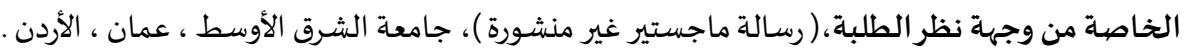

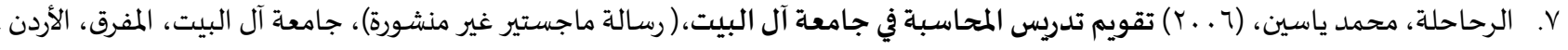

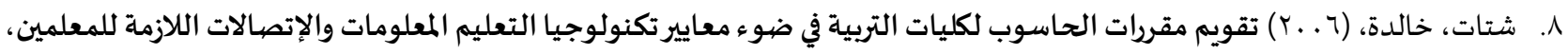

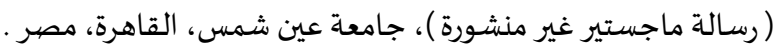

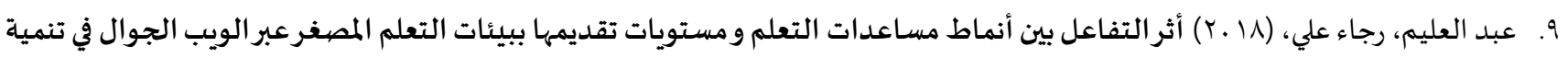

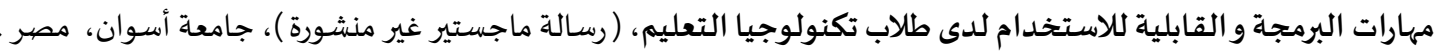

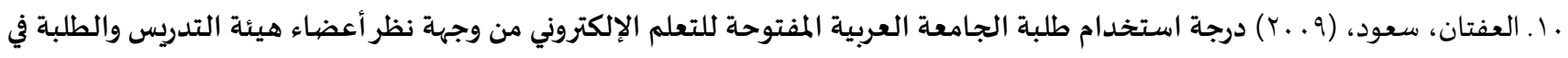

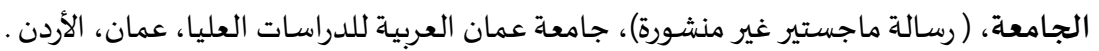

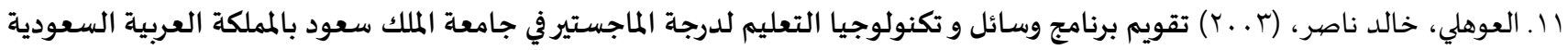

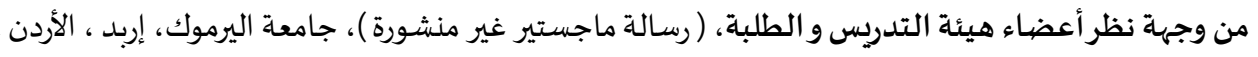

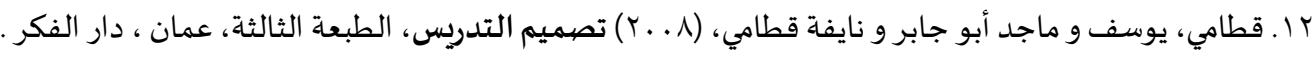

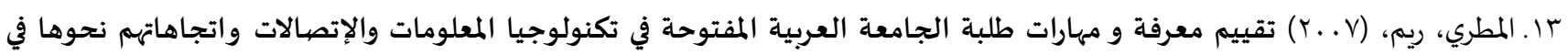

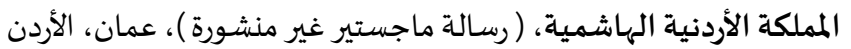

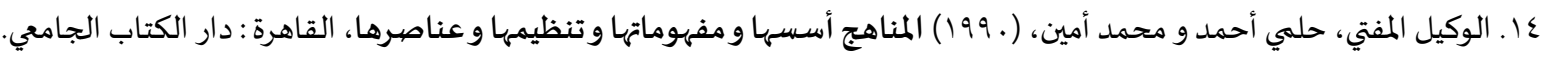

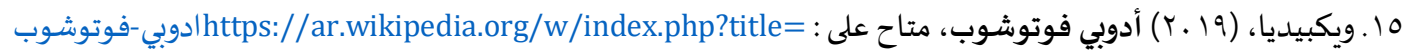

ثانياً: المراجع الأجنبية:

[1] Annison. jill, Action Research: Reviewing the Implementation of a Distance- Learning Degree Programme Utilizing Communication and Information Technologies, Innervtions in Education and Teaching International, 39(5) (2002), https://doi.org/10.1080/14703290252934540

[2] Kozma. Robert, Closing the Digital Divide evaluation of the World Links Program, International Journal of Education Development, 24 (4) (2004), https://doi.org/10.1016/s0738-0593(03)00155-x 
المجلة الدولية للدراسـات التربوية والنفسية

International Journal of Educational \& Psychological Studies (EPS)

Journal Homepage: https://www.refaad.com/views/EPSR/Home.aspx

www.refaad.com

ISSN: 2520-4149 (Online) 2520-4130 (Print)

\title{
The Performing Skills in the Graphic Design Course for Visual Media in the Field of Educational Technology for the Undergraduate Stage in the Middle East University and the Extent of Applying it from the Student's Point of View
}

\author{
Lina Rasem Arafeh \\ Computer Teacher, The Ministry of Education of Jordan \\ Lina3arafah84@gmail.com
}

Received Date : 18/11/2019

Accepted Date : $15 / 12 / 2020$

DOI : https://doi.org/DOI:10.31559/EPS2020.7.3.6

\begin{abstract}
This study aimed to determine the performance skills (practical) in the graphic design for visual media in the educational technology specialization for a bachelor's degree at the University of the Middle East using the content analysis tool, and to determine the extent of students 'application of the performance skills (practical) included in the course and applied using the program (Adobe Photoshop cc) from their point of view. The study sample consisted of (15) students, and the results of the study showed that the number of practical performance skills found in the course are (35) skills, and the extent of students' applying of it from their point of view has reached $79.68 \%$, with an arithmetic average of (3.98) which classified the application as a high degree. The researcher recommends focusing on activating and linking computer software to produce electronic educational content and analyzing more courses related to educational technology; identifying practical performance skills included in it. Where the researcher noted the scarcity of research in the field of analysis for the course of Education Technology in order to identify strengths points and identify weaknesses points to solve them, enhance the role of projects for the courses of the practical materials
\end{abstract}

Keywords: performance skills; courses; graphic design of the visual media; Adobe Photoshop cc; educational technology.

\section{References:}

[1] 'bd Al'lym, Rja' 'ly, Athr Altfa'l Byn Anmat Msa'dat Alt'lm W Mstwyat Tqdymha Bby'at Alt'lm Almsghr 'br Alwyb Aljwal Fy Tnmyh Mharat Albrmjh W Alqablyh Llastkhdam Lda Tlab Tknwlwjya Alt'lym, (Rsalt Majstyr Ghyr Mnshwrh), Jam't Aswan, Msr, (2018).

[2] Al'ftan, S'wd, Drjt Astkhdam Tlbt Aljam'h Al'rbyh Almftwhh Llt'lm Alelktrwny Mn Wjht Nzr A'da' Hy't Altdrys Waltlbh Fy Aljam'h, (Rsalt Majstyr Ghyr Mnshwrh), Jam't 'man Al'rbyh Lldrasat Al'lya, 'man, Alardn, (2009).

[3] Al'whly, Khald Nasr, Tqwym Brnamj Wsa'l W Tknwlwjya Alt'lym Ldrjt Almajstyr Fy Jam't Almlk S'wd Balmmlkh Al'rbyh Als'wdyh Mn Wjht Nzr A'da' Hy't Altdrys W Altlbh, (Rsalt Majstyr Ghyr Mnshwrh), Jam't Alyrmwk, Erbd, Alardn, (2003).

[4] Annison. jill, Action Research: Reviewing the Implementation of a Distance- Learning Degree Programme Utilizing Communication and Information Technologies, Innervtions in Education and Teaching International, 39(5) (2002), https://doi.org/10.1080/14703290252934540

[5] Ebrahym. Mna Taj Alsr, Tqwym Mqrr Altsmym Alt'lymy Lmajstyr Tknwlwjya Alt'lym Bklyt Altrbyh- Jam't Alswdan Ll'lwm W Altknwlwjya Fy Dw' M'ayyr Aljwdh Alshamlh, (Rsalt Majstyr Ghyr Mnshwrh), Jam't Alswdan Ll'lwm Waltknwlwjya Alt'lym, Alkhrtwm, Alswdan, (2016).

[6] Eshtwh. Fwzy Fayz \& 'lyan. Rbhy Mstfa, Tknwlwjya Alt'lym (Alnzryh W Almmarsh), 'man: Dar Almsyrh Llnshr W Altwzy', (2010). 
[7] Abw Alhaj. 'ta Ahmd Hsn, Drash Tqwymyh Lbrnamj Aldblwm Al'aly Fy Tknwlwjya Alm'lwmat Waletsalat Fy Altrbyh (Icte) Fy Aljam'h Alardnyh, (Atrwht Dktwrah Ghyr Mnshwrh), Aljam'h Alardnyh, 'man, Alardn, (2009).

[8] Alhyary. Yasmyn "Mhmd Nwr" 'bd Alrzaq, Tqwym Mqrrat Tkhss Tknwlwjya Alt'lym Lmrhlh Albkalwryws Fy Aljam'at Alardnyh Alkhash Mn Wjht Nzr Altlbh, (Rsalt Majstyr Ghyr Mnshwrh), Jam't Alshrq Alawst, 'man, Alardn, (2017).

[9] Jam't Alshrq Alawst, Khtt Aldrash, Klyh Al'lwm Altrbwyh, (2019), Mtah 'la: https://meu.edu.jo/alklyat/klyhal'elwm-altrbwyh/khtt-aldrash

[10] Jam't Alshrq Alawst, Rsalt Al'myd, Klyt Al'lwm Altrbwyh, (2019), Mtah 'Ela: https://meu.edu.jo/alklyat/klyhal'elwm-altrbwyh/rsalh-al'emyd

[11] Kozma. Robert, Closing the Digital Divide evaluation of the World Links Program, International Journal of Education Development, 24 (4) (2004), https://doi.org/10.1016/s0738-0593(03)00155-x

[12] Almtry.Rym, Tqyym M'rfh W Mharat Tlbt Aljam'h Al'rbyh Almftwhh Fy Tknwlwjya Alm'lwmat Waletsalat Watjahathm Nhwha Fy Almmlkh Alardnyh Alhashmyh, (Rsalh Majstyr Ghyr Mnshwrh), 'man, Alardn, (2007).

[13] Qtamy. Ywsf, Majd Abw Jabr \& Nayfh Qtamy, Tsmym Altdrys, Altb'h Althalthh, 'man, Dar Alfkr, (2008).

[14] Alrhahlh. Mhmd Yasyn, Tqwym Tdrys Almhasbh Fy Jam't Al Albyt, (Rsalt Majstyr Ghyr Mnshwrh), Jam't Al Albyt, Almfrq, Alardn, (2006)

[15] Shtat. Khaldh, Tqwym Mqrrat Alhaswb Lklyat Altrbyh Fy Dw' M'ayyr Tknwlwjya Alt'lym Alm'lwmat Waletsalat Allazmh Llm'Imyn, (Rsalt Majstyr Ghyr Mnshwrh), Jam't 'yn Shms, Alqahrh, Msr, (2006).

[16] Alwkyl Almfty. Hlmy Ahmd \& Mhmd Amyn, Almnahj Assha W Mfhwmatha W Tnzymha W 'nasrha, Alqahrh : Dar Alktab Aljam'y, (1990).

[17] Wykbydya, Adwby Fwtwshwb, (2019), Mtah 'la: https://ar.wikipedia.org/w/index.php?title=adwbyfutwshwb 